\title{
Preface
}

2015 3rd Asia Conference on Mechanical and Materials Engineering (ACMME 2015) was held in Mechanical and Materials Engineering during July 23-24, 2015. The conference provides a platform to discuss Mechanical and Materials Engineering etc. with participants from all over the world, both from academia and from industry. Its success is reflected in the papers received, with participants coming from several countries, allowing a real multinational multicultural exchange of experiences and ideas.

The present volumes collect accepted papers and represent an interesting output of this conference. This book covers these topics: Advanced materials and properties, Material processing and preparation technology, Mechanical design and manufacturing, Mechatronics and Robotics.

After the peefreview process, the submitted papers were selected on the basis of originality, significance, and clarity for the purpose of the conference. The selected papers and additional late-breaking contributions to be presented as lectures will make an exciting technical program. The conference program is extremely rich, featuring high-impact presentation. We hope that the conference results constituted significant contribution to the knowledge in these up to date scientific field.

The proceedings records the fully refereed papers presented at the conference. The main conference themes and tracks are Mechanical and Materials Engineering etc. Hopefully, all participants and other interested readers benefit scientifically from the proceedings and also find it stimulating in the process.

This conference can only succeed as a team effort, so the editors want to thank the international scientific committee and the reviewers for their excellent work in reviewing the papers as well as their invaluable input and advice.

\section{Jasmine Zhou}

\section{Hao Gong}

